\title{
A multi-approach management intervention can lower C-section rate trends: The experience of a Third Level Referral Center
}

\author{
de Belvis A.G. ${ }^{1,2}$, Neri C. ${ }^{* 1}$, Angioletti C. ${ }^{1}$, Carducci B. ${ }^{1}$, Ferrazzani S. ${ }^{1,3}$, Lanzone A. ${ }^{1,3}$, Caruso A. ${ }^{1,3}$ \\ ${ }^{1}$ Fondazione Policlinico Universitario A. Gemelli IRCCS, Roma, Italy \\ ${ }^{2}$ Section of Hygiene - Institute of Public Health; Università Cattolica del Sacro Cuore, Roma, Italy \\ ${ }^{3}$ Department of Obstetrics and Gynecology, Università Cattolica del Sacro Cuore, Roma, Italy
}

Received: October 6, 2018

Accepted: November 14, $2018 \quad$ Online Published: November 19, 2018

DOI: $10.5430 /$ jha.v7n6p37

URL: https://doi.org/10.5430/jha.v7n6p37

\begin{abstract}
Objective: To report the experience developed in a Third Level Referral Center in performing a multifaceted intervention strategy to reduce Caesarian Sections (CS) rate. A comparison of our results with the performance of the best Italian hospitals for number of deliveries and CS has been performed.

Methods: A monitoring system was set up, based on a prospective collection of all deliveries from 2013 to 2017, according to Robson's classification. Data have also been collected retrospectively at a regional and national level to compare our results to other institutions. The multi-approach intervention consisted of evidence based tools: process management, training, multiprofessionalism, development of planning and control systems, continuous monitoring, audit and feedback.

Results: The percentage of primary CS decreased from $26.71 \%$ in 2013 to $15.03 \%$ in 2017 (RR adjusted considering the regional average: 0.87 in 2013; 0.57 in 2017, $p<.001$ ). A raise of $19.76 \%$ in the annual volume of deliveries was registered. Such results have also been confirmed after comparing to the best performing Italian centers. From 2013 to 2016 the percentage of primary CS decreased from $27.02 \%$ to $18.04 \%$ (RR adjusted considering the regional average: 1.04 in 2013, $p>.05 ; 0.74$ in 2016 , $p<.001$ ), while there was an increase in the annual volume of deliveries from 3,311 to 4,219.

Conclusions: Our study confirms that multifaceted interventions can strengthen a continuous quality and safety improvement approach. This is of crucial relevance in the obstetric field and in the Italian country, where overall performance in CS needs to be improved.
\end{abstract}

Key Words: C-section rate, Patient centeredness, Multifaceted intervention, Quality improvement, Hospital management

\section{INTRODUCTION}

In the last few decades, specifically since 1970, a major public health problem has been the continuous increase of Caesarian Sections (CS) rates, both in the developed and developing countries. ${ }^{[1]}$

In 1985, the World Health Organization (WHO) indicated that a CS rate greater than $10 \%-15 \%$ was not justified for any region of the world to ensure optimal maternal and perinatal outcomes, ${ }^{[2]}$ while some recent revisions support an optimal global international CS rate of $19 \% .^{[3]}$

C-section is one of the most frequently performed surgical procedures $^{[4]}$ and is one of those with greater impact for the

*Correspondence: Neri C.; Email: caterina.neri@policlinicogemelli.it; Address: Fondazione Policlinico Universitario A. Gemelli IRCCS, Largo A. Gemelli 8, 00168 Roma, Italy. 
National Health System ${ }^{[5]}$ in terms of clinical inappropriateness and waste of resources. ${ }^{[6]}$

According to the Organization for Economic Co-operation and Development Data ${ }^{[7]}$ - among European countries - Italy, Poland, and Hungary have the highest CS rate $(35.7 \%)$, whereas Scandinavian nations have the lowest (Finland 15.8\%; Norway 16.6\%; and Sweden 17.0\%). Among Italian regions, Campania has the highest rate of CS (58.4\%).

At the beginning of this phenomenon, the wide introduction into clinical practice of cardiotocography (CTG) played a crucial role in the increase of unnecessary obstetric interventions. It is now clear that CTG has well-documented limitations, and it is necessary to be aware of these for safe use of the technology. It has been demonstrated that CTG analysis is subject to considerable intra- and inter-observer disagreement, even when experienced clinicians use widely accepted guidelines. ${ }^{[8-10]}$

Many studies have evaluated the ability of suspicious and pathological CTGs to predict the occurrence of neonatal hypoxia/acidosis. It is recognized that hypoxia/acidosis has not been documented shortly after a normal CTG tracing. On the other hand, suspicious and pathological tracings have a limited capacity to predict metabolic acidosis and low Apgar scores, i.e. a large percentage of cases with suspicious and pathological tracings do not have these outcomes. ${ }^{[1]}$

Moreover, continuous CTG was associated with a $63 \%$ increase in cesarean delivery and a $15 \%$ increase in instrumental vaginal deliveries, ${ }^{[12]}$ also due to its inappropriate introduction and use into legal medical issues.

Possible causes for the increase of CS rates may also include progressive modifications in socio-demographic factors, as well as changes in the attitudes of health professionals towards legal medical issues and different approaches of women towards pain and labor.

In the recent years, several approaches have been proposed, and some of them introduced into clinical practice, in order to reduce CS rate in those countries where an effort was requested by the local health management system.

The method chosen by the WHO as the most appropriate one to satisfy international and local needs is a classification system of 10 classes of women admitted for delivery, divided into five obstetric groups. This system is named by its author as the Robson 10-Group Classification System (RTGCS) (see Table 1). ${ }^{[5,13,14]}$ Since its introduction, RTGCS has gained a wide acceptance, as it allows to compare interinstitutional characteristics and behaviors in the same nation and among different nations, and it helps to introduce and promote easy-to-implement strategies to reduce CS rates. ${ }^{[15]}$

Moreover, the dictum "once a cesarean always a cesarean" also partly contributed to the increase in the rate of cesarean deliveries. ${ }^{[16]}$ However, in the 1970 s, some investigators began to reconsider this paradigm, and accumulated data have since supported Trial of Labor after Caesarian (TOLAC) as a reasonable approach in selected pregnancies. ${ }^{[17,18]}$ Recommendations favoring TOLAC were reflected in increased Vaginal Birth after Caesarian (VBAC) rates (VBAC per 100 women with a prior cesarean delivery) from slightly more than $5 \%$ in 1985 to $28.3 \%$ by 1996 . Concomitantly, the overall cesarean delivery rate decreased from $22.8 \%$ in 1989 to approximately $20 \%$ by 1996 [ $^{[19]}$ In 2010 , the National Institutes of Health convened a consensus conference to examine the safety and outcomes of TOLAC and VBAC as well as factors associated with their decreasing rates. The National Institutes of Health panel recognized that TOLAC was a reasonable option for many women with a prior cesarean delivery ${ }^{[4]}$ and called on organizations to facilitate access to TOLAC. ${ }^{[20]}$

Table 1. Robson 10-Group Classification System

\begin{tabular}{ll}
\hline Group & Robson 10-Classification System \\
\hline Group 1 & Nullipara, $\geq 37$ weeks, single, cephalic presentation, spontaneous labour. \\
Group 2 & Nullipara, $\geq 37$ weeks, single, cephalic presentation, induced labour or CS before labour. \\
Group 3 & Multipara, single, no previous CS, $\geq 37$ weeks, cephalic presentation, spontaneous labour. \\
Group 4 & Multipara, no previous CS, $\geq 37$ weeks, single, cephalic presentation, induced labour or CS before labour. \\
Group 5 & Multipara, previous CS, $\geq 37$ weeks, single, cephalic presentation. \\
Group 6 & Nullipara, single breech presentation. \\
Group 7 & Multipara, single breech presentation. \\
Group 8 & Multiple gestation (with or without previous CS). \\
Group 9 & Singleton pregnancy, oblique or transverse lies (excluding breech, with or without previous CS). \\
Group 10 & Singleton, cephalic pregnancy, <37 weeks (including previous CS). \\
\hline
\end{tabular}


In addition to this, recent evidences support the possibility to offer women, in selected cases, external cephalic version in case of breech presentation at term. ${ }^{[21]}$

In this scenario, in 2006, the Italian Region - Lazio where our institution Fondazione Policlinico Universitario A. Gemelli (FPG) - IRCCS (Istituto di Ricovero e Cura a Carattere Scientifico) - is located, introduced a list of performance indicators based on quality standards. These indicators are driven by strong clinical recommendations and aim to ensure the quality of health care organization, improving safety of care and helping health managers to understand how change occurs, in order to develop strategies that lead to innovation. ${ }^{[22]}$

A systematic review of the effectiveness of strategies adopted to reduce CS rate has shown that it can be safely reduced through multifaceted interventions that involve health workers in analyzing and modifying their practice, taking into account the clinical practice guidelines (CPGs). ${ }^{[23]}$
Starting from March 2013 and still ongoing, FPG defined and implemented a multifaceted intervention of change management to reverse a national and global inclination to perform CS to guarantee more safety in deliveries. The aim of this study is to evaluate such an intervention on the CS trend between 2013 and 2017. In addition, we seek to compare our results with the performance of the best Italian hospitals on number of deliveries and CS rates, by adjusting for case complexity.

\section{Methods}

To perform the analysis, data were collected from different sources at a local, regional and national level.

Locally, a prospective collection of all deliveries and a stratification of deliveries in 10-classes Robson classification assigned by a gynecologist working in the obstetric area, have been performed, building up a hospital monitoring system starting from 2013 (see Figure 1).

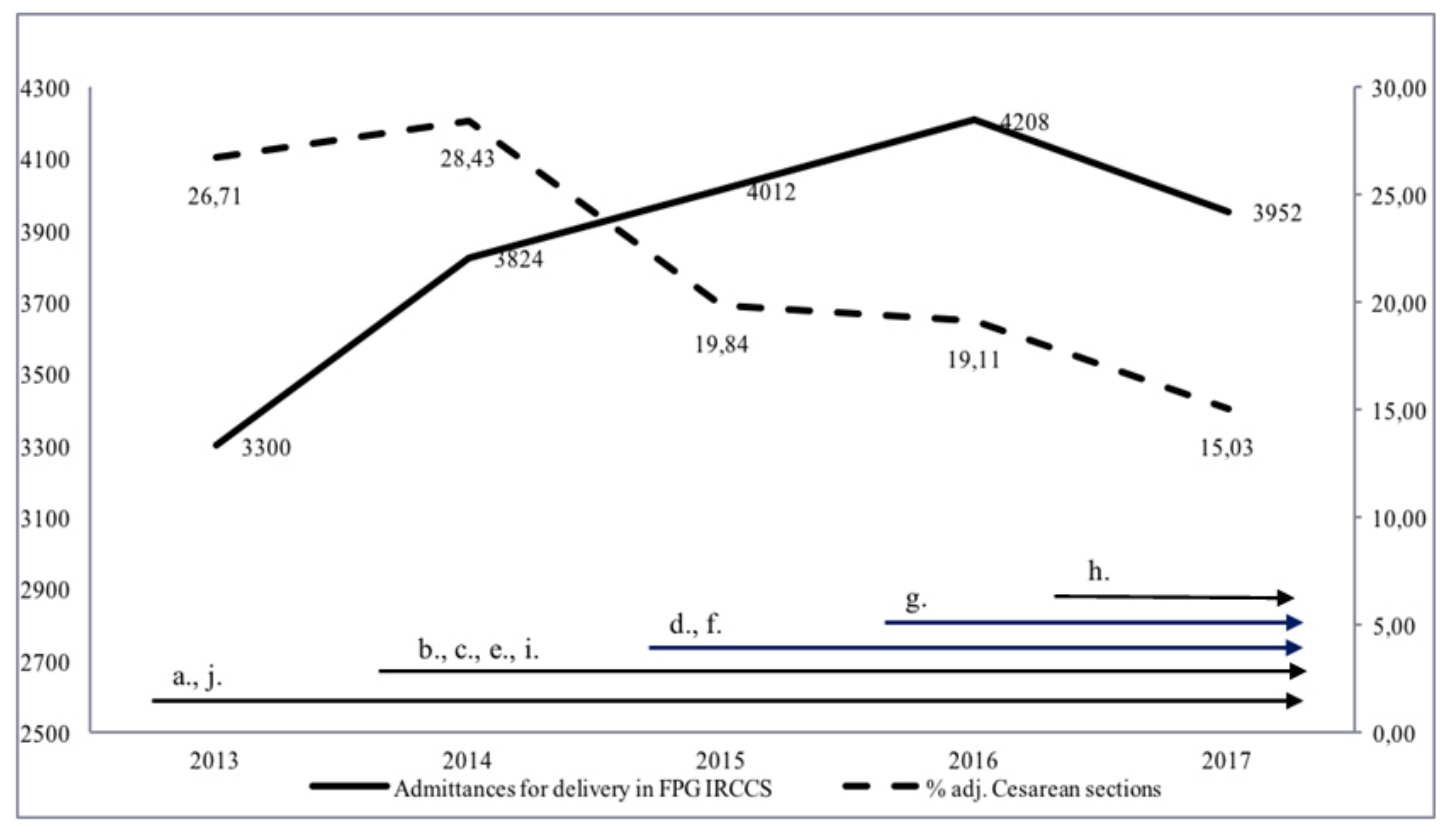

Figure 1. Primary CS adjusted rate and admittances for delivery in FPG IRCCS

Educational interventions and protocols

a. Introduction of External Cephalic Version (ECV) protocol

b. Professional training on Critical Pathways

c. Introduction of Oral Misoprostol in pre-labor rupture of membranes

d. Management of Obstetrical counseling before delivery and after a Cesarean section (TOLAC protocol)

Structural intervention

e. Emergency Area dedicated to Pregnancy

f. Turnover of the workforce in the Delivery Area

g. New leader in the role of the Obstetrician midwifery

h. 24 h presence of midwives in High Risk Pregnancy Unit

Organizational intervention

i. Implementation of the Clinical Pathway (C.P.) and multidisciplinary team

j. Monitoring System on Robson Groups, Audit and Feedback

Published by Sciedu Press 
At a regional level, data on deliveries and primary CS were retrospectively collected using P.Re.Val.E (Regional Evaluation Program, Programma Regionale Valutazione Esiti) web site (http://95.110.213.190/prevale2018/i ndex . php) based on the regional Structure Information Systems (HIS) database. The P.Re.Val.E. information system enrolled all discharged women that had a delivery in any structure within Lazio region between January 1st, 2013 and December 31th, 2017 (DRG 370-375, or ICD-9-CM primary or secondary diagnosis related group V27.xx or 640.xy-676.xy were $y=1$ or 2 , or procedure code 72.x, 73.2, 73.5, 73.6, 73.8, 73.9, 74.0, 74.1, 74.2, 74.4, 74.99, see Table 2). All deliveries were selected using Diagnosis-Related Groups (DRGs), diagnosis, and procedure International Classification of Diseases, Ninth Revision, Clinical Modification codes (ICD-9-CM).

Table 2. DRG and ICD-9-CM primary or secondary diagnosis related group

\begin{tabular}{ll}
\hline Codes & Description \\
\hline $370-375$ & C-section or vaginal delivery \\
V27.xx & Outcome of delivery \\
640.xx-648.xx & Complications mainly related to pregnancy \\
$650 . x x-659 . x x$ & Normal delivery, and other indications for care in pregnancy, labor, and delivery \\
660.xx-669.xx & Complications occurring mainly in the course of labor and delivery \\
$670 . x x-677 . x x$ & Complications of the puerperium \\
$72 . x, 73.2,73.5,73.6,73.8,73.9,74.0$, & Obstetrical procedures \\
$74.1,74.2,74.4,74.99$ & \\
\hline
\end{tabular}

The extracted codes were assigned for each patient by a physician working into the obstetric area, considering the medical records of the clinical chart at the time of patient discharge. More detailed information on the exclusion criteria used for the selection of the cohorts are available on the P.Re.Val.E. web site (http://95.110.213.190/prevale 2018/Main/protocolli/pro_37.pdf).

At a national level, to analyze the same indicators on the best performing Italian Hospitals by adjusting for case complexity, the PNE (National Evaluation Program, Programma Nazionale Esiti) data base was investigated. More detailed information on the exclusion criteria used for the selection of the cohorts are available on the PNE web site (http://pne2017.agenas.it/). The best Italian hospitals in the Obstetric Area have been chosen among Teaching, Hospital Trust, and other Third Level Referral Centers with over 600 beds.

The multi approach intervention consisted on evidence based tools (process management, training, multi-professionalism and multidisciplinary, development of planning and control systems, process monitoring, audit and feedback). Global awareness on $\mathrm{C}$-section reduction and a new leadership to steer the change management were introduced in 2013. Institutional guidelines to improve quality and safety in deliveries were then defined and implemented since 2014. They addressed several quality and safety topics, form outpatient care to post-partum follow up. In particular, an updated interpretation of intrapartum CTG was introduced based on the algorithm proposed by Clark et al. in 2013. ${ }^{\text {[24] }}$ This specific intervention gave clinicians the possibility to evaluate CTG tracings using an easier classification of CTG patterns (http://intranet.policlinicogemelli.it /download/lg-004_linee-guida-interne-sulla-n ascita_19062014-2/?wpdmdl=36452).

To evaluate its impact on care, a monitoring and feedback system based on the Robson classification has been implemented. In addition, monthly clinical audit meetings were systematically performed from March 2013. Since then, multiple protocols and interventions have been introduced and implemented in order to reduce $\mathrm{C}$-section rate, such as External Cephalic Version in selected cases of breech presentation, Trial Of Labor After C-Section in selected cases, the use of oral Misoprostol for induction of labor in pre-labor rupture of membranes (Gazzetta Ufficiale n. 261 November 10, 2014 - Agenzia Italiana del Farmaco), and the reinforcement of the Obstetric Area with gynecologists exclusively dedicated and fully trained into the obstetric field, according to the change management tools.

Each multifaceted intervention was implemented by a multidisciplinary team, where healthcare managers, obstetrics, midwives, anesthesiologists and neonatologists actively participated in a shared path, suitable for our institution, after applying scientific evidence.

All clinical and administrative interventions adopted from 2013 to reduce CS rate and improve health care assistance have been evaluated and listed as shown in Figure 1. 


\section{Results}

Between 2013 and 2017 the frequency of primary CS decreased from $26.71 \%$ to $15.03 \%$. This decrease in CS rate involves deliveries classified according to Robson classes with a lower clinical risk (e.g., Robson Group 1, which was associated with lower risk of potential complications peripartum; albeit with a lower rate, such a decrease was also registered among Robson Group 2a) (see Table 3).

Table 3. Cesarean delivery reduction in Robson Classes 1 and 2a

\begin{tabular}{lllllll}
\hline & $\mathbf{2 0 1 2}$ & $\mathbf{2 0 1 3}$ & $\mathbf{2 0 1 4}$ & $\mathbf{2 0 1 5}$ & $\mathbf{2 0 1 6}$ & $\mathbf{2 0 1 7}$ \\
\hline N patients Class 2A & 269 & 583 & 701 & 841 & 1,003 & 1,001 \\
\% CS & 18.6 & 29.7 & 31.2 & 17.8 & 14 & 13.7 \\
N patients Class 1 & 951 & 663 & 869 & 812 & 802 & 631 \\
\% CS & 24.9 & 17.5 & 15.2 & 11.0 & 10.1 & 8.6 \\
\hline
\end{tabular}

In the meanwhile, the annual volume of deliveries raised of $19.76 \%$ - from 3,300 to 3,952 (RR - Relative Risk - adjusted considering the regional average: 0.87 in 2013 with a $p$-value <.001; 0.57 in 2017 with a $p$-value < .001) (see Figure 1). Such a trend was also confirmed by comparing data on deliveries and primary CS with the most performing
Italian hospitals from 2013 to 2016 (see Tables 4-5). In the interval time considered, the percentage of primary CS decreased from $27.02 \%$ to $18.04 \%$ (RR adjusted considering the regional average: 1.04 in 2013 with a $p$-value $>.05 ; 0.74$ in 2016 with a $p$-value $<.001$ ), while there was an increase in the annual volume of deliveries - from 3,311 to 4,219.

Table 4. Admittance for delivery according to PNE data among the best performing Italian hospitals in the Obstetric Area

\begin{tabular}{lllll}
\hline Structure & $\mathbf{2 0 1 3}$ & $\mathbf{2 0 1 4}$ & $\mathbf{2 0 1 5}$ & $\mathbf{2 0 1 6}$ \\
\hline Structure 1 & 7,714 & 7,497 & 7,260 & 7,052 \\
Structure 2 & 6,245 & 6,130 & 6,210 & 5,906 \\
Structure 3 & 4,373 & 4,339 & 4,449 & 4,441 \\
Structure 4 & 3,749 & 3,909 & 4,134 & 4,235 \\
Fondazione Policlinico A. Gemelli IRCCS & 3,311 & 3,832 & 4,023 & 4,219 \\
Structure 6 & 3,635 & 3,776 & 3,645 & 3,565 \\
Structure 7 & 3,585 & 3,452 & 3,427 & 3,348 \\
Structure 8 & 3,530 & 3,544 & 3,626 & 3,234 \\
Structure 9 & 3,472 & 3,356 & 3,208 & 3,115 \\
Structure 10 & 2,493 & 2,585 & 2,672 & 2,695
\end{tabular}

Table 5. Primary CS adjusted rate according to PNE data among the best performing Italian hospitals in the Obstetric Area

\begin{tabular}{lllll}
\hline Structure & $\mathbf{2 0 1 3}$ & $\mathbf{2 0 1 4}$ & $\mathbf{2 0 1 5}$ & $\mathbf{2 0 1 6}$ \\
\hline Structure 1 & 7,714 & 7,497 & 7,260 & 7,052 \\
Structure 2 & 6,245 & 6,130 & 6,210 & 5,906 \\
Structure 3 & 4,373 & 4,339 & 4,449 & 4,441 \\
Structure 4 & 3,749 & 3,909 & 4,134 & 4,235 \\
Fondazione Policlinico A. Gemelli IRCCS & 3,311 & 3,832 & 4,023 & 4,219 \\
Structure 6 & 3,635 & 3,776 & 3,645 & 3,565 \\
Structure 7 & 3,585 & 3,452 & 3,427 & 3,348 \\
Structure 8 & 3,530 & 3,544 & 3,626 & 3,234 \\
Structure 9 & 3,472 & 3,356 & 3,208 & 3,115 \\
Structure 10 & 2,493 & 2,585 & 2,672 & 2,695 \\
\hline
\end{tabular}




\section{Discussion}

Our study showed a progressive reduction in the primary CS trends. Such a result bucks the trends of an increasing number of deliveries. We believe that a multifaceted change management approach played a crucial role in determining such improvements in clinical and obstetric care.

Interventions to address the main determinants of cesarean sections rates have been previously classified utilizing a combination of maternal, obstetric and organizational variables. ${ }^{[25]}$ Among these variables, physicians and their clinical behavior, as well as refund rates, play an important role in the Italian healthcare system, where appropriateness and universal coverage orient the distribution and the organization of the delivery system.

In our hospital it was evaluated that, through such multi management interventions and with the reduction of CS rate, an overall saving of about 400.000 euros has been achieved in the last two years.

It is important to underline the usefulness of the introduction of monitoring, audit and feedback together with updating meetings on guidelines, in particular those regarding the interpretation of CTG and the periodic revision of clinical cases ended with caesarian section.

Multi-disciplinary, multi-professional and trans-professional approach were guaranteed in all phases of the interventions: from priority setting to definition, from implementation to final appraisal.

In addition to this, the central role of the obstetric leadership allowed to ensure quality in health care and in coordination of all health professionals. This has also been possible af ter the introduction into the delivery room of gynecologists exclusively dedicated to the obstetric field, while midwives gained autonomy in managing low-risk cases. Moreover, oral misoprostol introduction in November 2014 brought to an evident improvement in the obstetric assistance and outcomes in those patients that needed induction for pre-labor rupture of membranes.

All these multifaceted interventions brought to fast improvement in health care into the obstetric field in our institution, according to what is reported in the literature.

In fact, all the interventions in our study were likely to show their robustness: a systematic review of the effectiveness of strategies for reducing CS has shown that CS rate can be safely reduced through multifaceted interventions that involve health workers in analyzing and modifying their practice, taking into account the CPGs. ${ }^{[26]}$ Educational intervention projects with health professionals have recently been implemented in Spain and Canada with the aim to decrease the number of preventable CS, leading to improved quality and patient safety. The projects hypothesize that poor adherence to CPGs plays a key role in the rising CS rate. ${ }^{[27]}$

The need to implement strategies to reduce CS rate started to arise in the 80 's. Some authors proposed a program that included a stringent requirement for a second opinion, objective criteria for the most common indications for cesarean section, and a detailed review of all cesarean sections and of individual physicians' rates of performing them. ${ }^{[28]}$ These authors demonstrated that an initiative within an obstetric department can reduce CS rates substantially without adverse effects on the outcome for mother or infant. Moreover, a cluster randomized controlled trial of 149.276 deliveries conducted in 36 hospitals in Latin America, where the CS rate is high, tested the hypothesis that a hospital policy of mandatory second opinion, based on the best existing scientific evidence, reduces the hospital caesarean section rate by $25 \%$, without increasing maternal and perinatal morbidity and mortality. ${ }^{[29]}$ In 2007, a meta-analysis of evidence-based strategies for reducing caesarian section rate including 10 studies, already highlighted that audit and feedback, quality improvement, and multifaceted strategies are effective in changing clinical practice and reducing caesarian section rates. ${ }^{[23]}$

Some limitations of this study must be acknowledged. One limitation is the partial retrospective collection of cases, but we believe that the large number of cases $(19,000$ deliveries from 2013 to 2017) and the clear evidence of reduction of CS rate (from $44 \%$ in 2013 to $34.7 \%$ in 2017) can reappraise this limitation.

In addition to this, describing association between exposure and outcome, without determining direct causation, is a limitation. Consequently, it is not possible to conclude that the multifaceted intervention or its sequence by itself led to a better outcome. Furthermore, content validity could be improved by adding qualitative indicator, such as patient's perspective, to assess improvements from multiple angles, as reported by Ansmann et al. ${ }^{[30]}$ These improvements are foreseen, but should still be implemented in our hospital monitoring system by introducing other metrics such as quality of life or functional status assessment.

As for the evaluation measures, in our study the national performance framework adopted in Italy according to PNE's was considered. Unfortunately, we could not adopt more detailed tools to examine the relationship between individual socioeconomic level, demographic factors and the utilization of a specific package of maternal health services, as Andersen's behavioral model of health service proposes. ${ }^{[31]}$ In 
fact, the Italian performance PNE database output does not provide this type of information (e.g. age, education level attainment, region of residence, type of place of residence, religion, marital status and number of children ever born).

On the other hand, we can consider as indirect quality indicator the increase in the number of deliveries from 3,255 in 2013 to 3,910 in 2017 with a peak of 4,148 deliveries in 2016.

Even considering the limitations of this cross-sectional study, our research is one of the first in Italy to evaluate the impact of a multi approach intervention to reduce the rate of unnecessary cesarean sections in a Third Level teaching hospital, and to demonstrate that such a reduction would match with an increase in the overall rate of births. Moreover, it is possible to underline how the introduction of the internal guidelines has been followed by a relevant decrease in primary CS, with obvious positive effects under the socio-economic profile.

In conclusion, this study shows how multifaceted interventions can strengthen the results achieved according to a continuous quality improvement approach that is crucial to guarantee safer and more effective care into the obstetric field, especially in a country, like Italy, whose overall performance in CS needs to be improved.

\section{CONFLicts OF INTEREST Disclosure}

The authors declare they have no conflicts of interest.

\section{REFERENCES}

[1] Betrán AP, Merialdi M, Lauer JA, et al. Rates of caesarean section: analysis of global, regional and national estimates. Paediatr Perinat Epidemiol. 2007; 21: 98-113. PMid: 17302638. https: //doi.org/10.1111/j.1365-3016.2007.00786.x

[2] World Health Organization. Appropriate technology for birth. Lancet. 1985; 2: 436-7. PMid: 2863457.

[3] Molina G, Weiser TG, Lipsitz SR, et al. Relationship between cesarean delivery rate and maternal and neonatal mortality. JAMA. 2015; 314: 2263-70. PMid: 26624825. https ://doi.org/10.100 $1 /$ jama. 2015.15553

[4] National Institutes of Health. Consensus Development conference statement: vaginal birth after cesarean: new insights. 2010.

[5] World Health Organization. WHO statement on caesarean section rates. Geneva. 2015

[6] Decreto del Commissario ad Acta n. U00412. Riorganizzazione della rete ospedaliera a salvaguardia degli obiettivi strategici di rientro dai disavanzi sanitari della Regione Lazio. Regione Lazio. 2014.

[7] OECD. Caesarean sections (indicator). June 2017.

[8] Paneth N, Bommarito M, Stricker J. Electronic fetal monitoring and later outcome. Clin. Invest. Med. 1993; 159-165. PMid: 8513616.

[9] Ayres-de-Campos D, Bernardes J, Costa-Pereira A, et al. Inconsistencies in classification by experts of cardiotocograms and subsequent clinical decision. BJOG. 1999; 1307-1310.

[10] Blackwell SC, Grobman WA, Antoniewicz L, et al. Interobserver and intraobserver reliability of the NICH 3-tier fetal heart rate interpretation system. Am. J. Obstet. Gynecol. 2011; 205(4): 378.e1-5. PMid: 21864826. https://doi.org/10.1016/j.ajog.2011.06.086

[11] Spencer JA. Clinical overview of cardiotocography. Br. J Obstet Gynaecol. 1993; 4-7. PMid: 8471569. https://doi.org/10.1111/ j.1471-0528.1993.tb10626.x

[12] Alfirevic Z, Devane D, Gyte GM. Continuous cardiotocography (CTG) as a form of electronic fetal monitoring (EFM) for fetal assessment during labour. Cochrane Database Syst Rev. 2013. https://doi.org/10.1002/14651858.CD006066.pub2

[13] Torloni MR, Betran AP, Souza JP, et al. Classifications for cesarean section: a systematic review. PLoS One. 2011; 6: e14566. PMid: 21283801. https://doi.org/10.1371/journal.pone.00145 66

Published by Sciedu Press
[14] Robson MS. Classification of caesarean sections. Fetal Matern Med Rev. 2011; 12: 23-39.

[15] Brennan DJ, Robson MS, Murphy M, et al. Comparative analysis of international cesarean delivery rates using 10-group classification identifies significant variation in spontaneous labor. Am J Obstet Gynecol. 2009; 201: 308.e1-8. PMid: 19733283. https: //doi.org/10.1016/j.ajog.2009.06.021

[16] Cragin EB. Conservatism in obstetrics. NY Med J. 1916; 104: 1-3.

[17] Landon MB, Hauth JC, Leveno KJ, et al. Maternal and perinatal outcomes associated with a trial of labor after prior cesarean delivery. N Engl J Med. 2004; 351: 2581-9. PMid: 15598960. https ://doi.org/10.1056/NEJMoa040405

[18] Macones GA, Peipert J, Nelson DB, et al. Maternal complications with vaginal birth after cesarean delivery: a multicenter study. Am J Obstet Gynecol. 2005; 193: 1656-62. PMid: 16260206. https://doi.org/10.1016/j.ajog.2005.04.002

[19] Menacker F, Declercq E, Macdorman MF. Cesarean delivery: background, trends, and epidemiology. Semin Perinatol. 2006; 30: 235 41. PMid: 17011392. https://doi.org/10.1053/j.semperi. 2006.07 .002

[20] Grobman W. Vaginal Birth After Cesarean Delivery. Committee on Practice Bulletins-Obstetrics. Obstet Gynecol. 2017; 130(5): e217e233

[21] Green-top Guideline. External Cephalic Version and Reducing the Incidence of Term Breech Presentation. BJOG. 2017; 124(7): e178e192. PMid: 28299867. https://doi.org/10.1111/1471-052 8.14466

[22] World Health Organization. Strategies for assisting health workers 2000.

[23] Chaillet N, Dumont A. Evidence Based Strategies for Reducing Cesarean Section Rates: A Meta-Analysis. Birth Issue in Perinatal Care. 2007; 53-64. PMid: 17324180.

[24] Clark SL, Nageotte MP, Garite TJ, et al. Intrapartum management of category II fetal heart rate tracings: towards standardization of care. American Journal Obstet Gynecol. 2013. https : //doi.org/10.1016/j.ajog.2013.04.030

[25] Stivanello E. Determinants of cesarean delivery: a classification tree analysis. Bio Med Central. 2014. 
[26] Bermúdez-Tamayo C, Johri M, Perez-Ramos FJ, et al. Evaluation of quality improvement for cesarean sections programmes through mixed methods. Bio Med Central. 2014.

[27] Aarons GA, Green AE, Willging CE, et al. Mixed-method study of a conceptual model of evidence-based intervention sustainment across multiple public-sector service settings. Implementation Science. 2014. https : //doi .org/10.1186/s13012-014-0183-z

[28] Myers SA, Gleicher N. A successful program to lower cesareansection rates. N Engl J Med. Dec 1988; 319(23): 1511-6. PMid: 3185675. https://doi.org/10.1056/NEJM198812083192304

[29] Althabe F, Belizán JM, Villar J, et al. Latin American Caesarean
Section Study Group. Mandatory second opinion to reduce rates of unnecessary caesarean section in Latin America: a cluster randomised controlled trial. Lancet. Jun 2004; 363(9425): 1934-40. https://doi.org/10.1016/S0140-6736(04)16406-4

[30] Ansmann L, Pfaff H. Providers and Patients Caught Between Standardization and Individualization: Individualized Standardization as a Solution Comment on "( $\mathrm{Re})$ Making the Procrustean Bed? Standardization and Customization as Competing Logics in Healthcare". International Journal of Health Policy Management. 2018; 349-352.

[31] Andersen RM. Revisiting the behavioural model and access to medical care: does it matter? J Health Soc Behav. Mar 1995; 36(1): 1-10. PMid: 7738325. https://doi.org/10.2307/2137284 